\title{
Higher order invariants in the case of compact quotients
}

\author{
Anton Deitmar \\ Central European Journal of Mathematics 9.1, 85-101 (2011)
}

\begin{abstract}
We present the theory of higher order invariants and higher order automorphic forms in the simplest case, that of a compact quotient. In this case many things simplify and we are thus able to prove a more precise structure theorem than in the general case.
\end{abstract}

\section{Contents}

1 Higher order invariants 2

2 Higher order cohomology 4

3 Hecke pairs and smooth modules $\quad 7$

4 Higher order $L^{2}$-spaces $\quad 10$

5 Lie groups 14

6 Sheaf cohomology 16

$\begin{array}{llr}7 & \text { Cohomology of lattices in Lie groups } & 19\end{array}$

8 Arithmetic groups $\quad 21$ 


\section{Introduction}

Higher order modular forms show up in various contexts, for instance in percolation theory [16], or in the theory of Eisenstein-series twisted by modular symbols $[2,12,13]$. Finally, spaces of higher order forms are natural receptacles of converse theorems $[10,15]$.

L-functions of second order forms have been studied in [7], Poincaré series attached to higher order forms have been investigated in [14], dimensions of spaces of second order forms have been determined in $[8,9]$. Higher order cohomology has been introduced and an Eichler-Shimura type theorem has been proven in [4]. In [5] a program has been started which aims at an understanding of the theory of higher order forms from an representationtheoretical point of view.

The paper [6] contains a structure theorem showing that spaces of higher order automorphic forms are in a natural way subspaces of tensor products of automorphic representation spaces. In this paper we restrict to the simple case of a compact quotient in which case we are able to

- remove the "subspace" part from the assertion, i.e., get a more precise statement on the structure of higher order forms, and

- extend the theory to $L^{2}$-invariants instead of smooth invariants only.

Also, this presentation has the advantage of being much simpler than in the general setting, for instance, in the case of compact quotient, there is no need to factor out cuspidal ideals and so all formulae look simpler.

\section{Higher order invariants}

Let $R$ be a commutative ring with unit. In the main applications, $R$ will be the field of complex numbers. Let $\Gamma$ be a group and let $I$ denote the augmentation ideal in the group algebra $A=R[\Gamma]$. For an $A$-module $V$ we define the $R$-module

$$
\mathrm{H}_{q}^{0}(\Gamma, V)
$$

of invariants of order $q \geq 1$ to be the set of all $v \in V$ with $I^{q} v=0$. Note that for $q=1$ one gets the usual invariants

$$
\mathrm{H}_{1}^{0}(\Gamma, V)=\mathrm{H}^{0}(\Gamma, V)=V^{\Gamma} .
$$


There is a natural identification

$$
\mathrm{H}_{q}^{0}(\Gamma, V) \cong \operatorname{Hom}_{A}\left(A / I^{q}, V\right) .
$$

The sets $\mathrm{H}_{q-1}^{0}(\Gamma, V) \subset \mathrm{H}_{q}^{0}(\Gamma, V)$ form a filtration on $V$ which is not necessarily exhaustive. Let

$$
\overline{\mathrm{H}}_{q}^{0}(\Gamma, V) \stackrel{\text { def }}{=} \mathrm{H}_{q}^{0}(\Gamma, V) / \mathrm{H}_{q-1}^{0}(\Gamma, V)
$$

be the $q$-th graded piece, where we allow $q=1,2, \ldots$ by formally setting $\mathrm{H}_{0}^{0}(\Gamma, V)=0$.

For an abelian group $G$, let $\operatorname{Hom}(\Gamma, G)$ denote the set of all group homomorphisms $\Gamma \rightarrow G$.

Lemma 1.1 Suppose that $R$ is a field. If $\Gamma$ is finitely generated as a group or if $W$ is finite-dimensional, then

$$
\operatorname{Hom}(\Gamma, W) \cong \operatorname{Hom}(\Gamma, R) \otimes W
$$

where the tensor product is over $R$.

Proof: This is easy to see.

Definition 1.2 We introduce the order-lowering-homomorphism

$$
\Lambda=\Lambda_{q}: \overline{\mathrm{H}}_{q}^{0}(\Gamma, V) \rightarrow \operatorname{Hom}\left(\Gamma, \overline{\mathrm{H}}_{q-1}^{0}(\Gamma, V)\right)
$$

given by

$$
\Lambda(v)(\gamma)=(\gamma-1) v
$$

To see that $\psi(v)$ is indeed a group homomorphism, note that in the group algebra $\mathbb{C}[\Gamma]$ one has $(\tau \gamma-1) \equiv(\tau-1)+(\gamma-1) \bmod I^{2}$.

Proposition 1.3 (a) The order-lowering operator $\Lambda$ is injective.

(b) If $R$ is a field and $\operatorname{Hom}(\Gamma, R)=\mathrm{H}^{1}(\Gamma, R)=0$, then there are no nontrivial higher order invariants, i.e., one has

$$
\mathrm{H}_{q}^{0}(\Gamma, V)=V^{\Gamma}
$$

for all $q \geq 1$. 
(c) If $R$ is a field and $\Gamma$ is finitely generated or $\operatorname{dim} V<\infty$, then the orderlowering operator induces an injective $\Gamma$-equivariant linear map

$$
\Lambda^{q}: \overline{\mathrm{H}}_{q}^{0}(\Gamma, V) \hookrightarrow \operatorname{Hom}(\Gamma, R)^{\otimes(q-1)} \otimes V^{\Gamma} .
$$

Proof: Part (a) is clear. Part (b) becomes clear by choosing a basis of $\bar{H}_{q-1}^{0}(\Gamma, V)$. The last assertion follows from Lemma 1.1 .

Lemma 1.4 Let $V$ be an $R[\Gamma]$-module which is torsion-free as $\mathbb{Z}$-module. Let $\Sigma \subset \Gamma$ be a subgroup of finite index. Then the natural restriction map

$$
\text { res : } \overline{\mathrm{H}}_{q}^{0}(\Gamma, V)^{0} \rightarrow \overline{\mathrm{H}}_{q}^{0}(\Sigma, V)
$$

is injective.

Note that the torsion-condition is automatic if $R$ contains the field $\mathbb{Q}$.

Proof: By induction on $q$.

\section{Higher order cohomology}

For an $R[\Gamma]$-module $V$ we define the higher order cohomology to be

$$
\mathrm{H}_{q}^{p}(\Gamma, V)=\operatorname{Ext}_{A}^{p}\left(A / I^{q}, V\right) .
$$

For $q=1$ this is ordinary group cohomology.

For an $R$-module $M$ and a set $S$ we write $M^{S}$ for the $R$-module of all maps from $S$ to $M$. Then $M^{\emptyset}$ is the trivial module 0 . Up to isomorphy, the module $M^{S}$ depends only on the cardinality of $S$. It therefore makes sense to define $M^{c}$ for any cardinal number $c$ in this way. Note that $I^{q} / I^{q+1}$ is a free $R$-module. Define

$$
N_{\Gamma}(q) \stackrel{\text { def }}{=} \operatorname{dim}_{R} I^{q} / I^{q+1} .
$$

Then $N_{\Gamma}(q)$ is a possibly infinite cardinal number.

Lemma 2.1 (a) For every $q \geq 1$ there is a natural exact sequence

$$
\begin{aligned}
& 0 \rightarrow H_{q}^{0}(\Gamma, V) \rightarrow H_{q+1}^{0}(\Gamma, V) \rightarrow H^{0}(\Gamma, V)^{N_{\Gamma}(q)} \rightarrow \\
& \rightarrow H_{q}^{1}(\Gamma, V) \rightarrow H_{q+1}^{1}(\Gamma, V) \rightarrow H^{1}(\Gamma, V)^{N_{\Gamma}(q)} \rightarrow \ldots \\
& \quad \cdots \rightarrow H_{q}^{p}(\Gamma, V) \rightarrow H_{q+1}^{p}(\Gamma, V) \rightarrow H^{p}(\Gamma, V)^{N_{\Gamma}(q)} \rightarrow \ldots
\end{aligned}
$$


(b) Suppose that for a given $p \geq 0$ one has $H^{p}(\Gamma, V)=0$. Then $H_{q}^{p}(\Gamma, V)=$ 0 for every $q \geq 1$. In particular, if $V$ is acyclic as $\Gamma$-module, then $H_{q}^{p}(\Gamma, V)=0$ for all $p \geq 1, q \geq 1$.

Note that $\mathrm{H}^{p}(\Gamma, V)^{N_{\Gamma}(q)}$ here comes up as $\operatorname{Ext}_{A}^{p}\left(I^{q} / I^{q+1}, V\right)$, which is important for the functoriality in the group $\Gamma$ of the above sequence.

Proof: Consider the exact sequence

$$
0 \rightarrow I^{q} / I^{q+1} \rightarrow A / I^{q+1} \rightarrow A / I^{q} \rightarrow 0 .
$$

As an $A$-module, $I^{q} / I^{q+1}$ is isomorphic to a direct sum $\bigoplus_{\alpha} R_{\alpha}$ of copies of $R=A / I$. So we conclude that for every $p \geq 0$,

$$
\operatorname{Ext}_{A}^{p}\left(I^{q} / I^{q+1}, V\right) \cong \prod_{\alpha} \operatorname{Ext}_{A}^{p}(R, V) \cong H^{p}(\Gamma, V)^{N_{\Gamma, \Sigma}(q)} .
$$

The long exact Ext-sequence induced by the above short sequence is

$$
\begin{aligned}
0 \rightarrow & \operatorname{Hom}_{A}\left(A / I^{q}, V\right) \rightarrow \operatorname{Hom}_{A}\left(A / I^{q+1}, V\right) \rightarrow \operatorname{Hom}_{A}\left(I^{q} / I^{q+1}, V\right) \rightarrow \\
& \rightarrow \operatorname{Ext}_{A}^{1}\left(A / I^{q}, V\right) \rightarrow \operatorname{Ext}_{A}^{1}\left(A / I^{q+1}, V\right) \rightarrow \operatorname{Ext}_{A}^{1}\left(I^{q} / I^{q+1}, V\right) \rightarrow \\
& \rightarrow \operatorname{Ext}_{A}^{2}\left(A / I^{q}, V\right) \rightarrow \operatorname{Ext}_{A}^{2}\left(A / I^{q+1}, V\right) \rightarrow \operatorname{Ext}_{A}^{2}\left(I^{q} / I^{q+1}, V\right) \rightarrow \ldots
\end{aligned}
$$

This is the claim (a). For (b) we proceed by induction on $q$. For $q=1$ the claim follows from $H_{1}^{p}(\Gamma, V)=H^{p}(\Gamma, V)$. Inductively, assume the claim proven for $q-1$ and $H^{p}(\Gamma, V)=0$. As part of the above exact sequence, we have the exactness of

$$
H_{q-1}^{p}(\Gamma, V) \rightarrow H_{q}^{p}(\Gamma, V) \rightarrow H^{p}(\Gamma, V)^{N_{\Gamma}(q)} .
$$

By assumption, we have $H^{p}(\Gamma, V)^{N_{\Gamma, \Sigma}(q)}=0$ and by induction hypothesis the module $H_{q-1}^{p}(\Gamma, V)$ vanishes. This implies $H_{q}^{p}(\Gamma, V)=0$ as well.

Lemma 2.2 For every $q \geq 1$ there is a natural isomorphism

$$
\operatorname{Hom}_{R}\left(I^{q} / I^{q+1}, R\right) \cong \mathrm{H}_{q}^{1}(\Gamma, R) .
$$

Proof: The exact sequence

$$
0 \rightarrow I^{q} \rightarrow A \rightarrow A / I^{q} \rightarrow 0
$$


induces an exact sequence

$0 \rightarrow \operatorname{Hom}_{A}\left(A / I^{q}, R\right) \stackrel{\alpha}{\longrightarrow} \operatorname{Hom}_{A}(A, R) \stackrel{\beta}{\longrightarrow} \operatorname{Hom}_{A}\left(I^{q}, R\right) \stackrel{\gamma}{\longrightarrow} \operatorname{Ext}_{A}^{1}\left(A / I^{q}, R\right) \rightarrow 0$.

Now $\alpha$ is an isomorphism, therefore $\beta$ is zero and $\gamma$ is an isomorphism again. We get an isomorphism

$$
\operatorname{Hom}_{R}\left(I^{q} / I^{q+1}, R\right) \cong \operatorname{Hom}_{A}\left(I^{q}, R\right) \cong \mathrm{H}_{q}^{1}(\Gamma, R) .
$$

Proposition 2.3 Suppose that $R$ is a field. Let $\Gamma$ be finitely generated and let $V$ be an $R[\Gamma]$-module with

$$
H^{1}(\Gamma, V)=0 .
$$

Then for every $q \geq 1$ there is a natural isomorphism

$$
\overline{\mathrm{H}}_{q+1}^{0}(\Gamma, V) \stackrel{\cong}{\longrightarrow} \mathrm{H}_{q}^{1}(\Gamma, R) \otimes V^{\Gamma} .
$$

Proof: By Proposition 2.1 we get an exact sequence

$$
0 \rightarrow \mathrm{H}_{q}^{0}(\Gamma, V) \rightarrow \mathrm{H}_{q+1}^{0}(\Gamma, V) \rightarrow \operatorname{Hom}_{R}\left(I^{q} / I^{q+1}, \mathrm{H}^{0}(\Gamma, V)\right) \rightarrow 0 .
$$

Since $\Gamma$ is finitely generated, the free $R$-module $I^{q} / I^{q+1}$ is finite-dimensional, hence we get

$$
\operatorname{Hom}_{R}\left(I^{q} / I^{q+1}, H^{0}(\Gamma, V)\right) \cong \operatorname{Hom}_{R}\left(I^{q} / I^{q+1}, R\right) \otimes H^{0}(\Gamma, V) .
$$

The claim now follows from Lemma 2.2 .

Lemma 2.4 For $q \geq 1$ the natural map

$$
\mathrm{H}_{q}^{1}(\Gamma, R) \rightarrow \mathrm{H}_{q+1}^{1}(\Gamma, R)
$$

is the zero map.

Proof: In Lemma 2.1 we put $V=R$ and get the exact sequence

$$
\operatorname{Hom}_{R}\left(I^{q} / I^{q+1}, R\right) \stackrel{\alpha}{\longrightarrow} \mathrm{H}_{q}^{1}(\Gamma, R) \rightarrow H_{q+1}^{1}(\Gamma, R) .
$$

We claim that $\alpha$ is an isomorphism. Note that $\alpha$ is the restriction of the connection morphism attached to the exact sequence

$$
0 \rightarrow I^{q} / I^{q+1} \rightarrow A / I^{q+1} \rightarrow A / I^{q} \rightarrow 0,
$$


which is related to the sequence

$$
0 \rightarrow I^{q} \rightarrow A \rightarrow A / I^{q} \rightarrow 0 .
$$

The connection morphism $\tilde{\alpha}$ of the latter was shown in Lemma 2.2 to be an isomorphism. Taking residue classes modulo $I^{q+1}$ maps the first exact sequence to the second. This results in a map of the corresponding long exact Ext-sequences, a part of which gives the commutative diagram

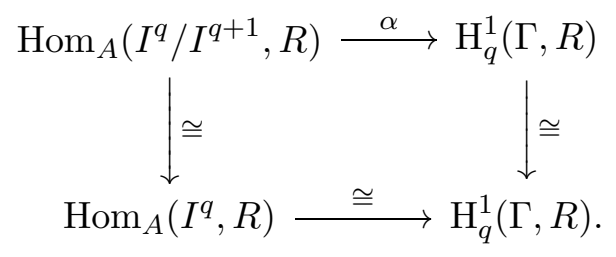

It follows that $\alpha$ is an isomorphism, indeed.

\section{$3 \quad$ Hecke pairs and smooth modules}

A Hecke pair is a pair $(\mathcal{G}, \Gamma)$ of a group $\mathcal{G}$ and a subgroup $\Gamma$ such that for every $g \in \mathcal{G}$ the set $\Gamma g \Gamma / \Gamma$ is finite. We also say that $\Gamma$ is a Hecke subgroup of $\mathcal{G}$.

Two subgroups $\Gamma, \Lambda$ of a group $H$ are called commensurable, written $\Gamma \sim \Lambda$, if the intersection $\Gamma \cap \Lambda$ has finite index in both. Commensurability is an equivalence relation which is preserved by automorphisms of $H$.

The commensurator of a group $\Gamma \subset H$ is

$$
\operatorname{comm}(\Gamma) \stackrel{\text { def }}{=}\left\{h \in H: \Gamma \text { and } h \Gamma h^{-1} \text { are commensurable }\right\} .
$$

Lemma 3.1 The commensurator $G=\operatorname{comm}(\Gamma)$ is a subgroup of $H$. It is the largest subgroup such that $(G, \Gamma)$ is a Hecke pair. More precisely,

$$
\operatorname{comm}(\Gamma)=\{h \in H:|\Gamma h \Gamma / \Gamma|,|\Gamma \backslash \Gamma h \Gamma|<\infty\} .
$$

Proof: Let $G=\operatorname{comm}(\Gamma)$ and $g \in G$. Then $\Gamma \sim g \Gamma g^{-1}$. By conjugating we get $g^{-1} \Gamma g \sim \Gamma$, hence $g^{-1} \in G$. Next let $h \in G$ as well. Then $\Gamma \sim h \Gamma h^{-1}$ and hence $g \Gamma g^{-1} \sim g h \Gamma(g h)^{-1}$ so that $\gamma \sim g \Gamma g^{-1}$ implies $\Gamma \sim g h \Gamma(g h)^{-1}$, which means that $g h \in G$, so $G$ is indeed a subgroup. 
To see the identity claimed in the lemma, one simply observes that for every $h \in H$ the natural map $\Gamma / \Gamma \cap h \Gamma h^{-1} \rightarrow \Gamma h \Gamma / \Gamma$, mapping the class of $\gamma$ to the class of $\gamma h$ is a bijection.

Let $\mathcal{G}$ be a group. By a $\mathcal{G}$-module we shall henceforth mean an $R[\mathcal{G}]$-module. If $\mathcal{G}$ is a totally disconnected topological group, an element $v$ of a $\mathcal{G}$-module $V$ is called smooth if it is stabilized by some open subgroup of the topological group $\mathcal{G}$. The set $V^{\infty}$ of all smooth elements is a submodule and the module $V$ is called smooth if $V=V^{\infty}$.

Drop the condition that $\mathcal{G}$ be a topological group and let $(\mathcal{G}, \Gamma)$ be a Heckepair. A congruence subgroup of $\Gamma$ is any subgroup which contains a group of the form

$$
\Gamma \cap g_{1} \Gamma g_{1}^{-1} \cap \cdots \cap g_{n} \Gamma g_{n}^{-1}
$$

for some $g_{1}, \ldots, g_{n} \in \mathcal{G}$. As $(\mathcal{G}, \Gamma)$ is a Hecke pair, every congruence subgroup has finite index in $\Gamma$. Note that this definition of a congruence subgroup coincides with the one given in [5]. For every congruence subgroup $\Sigma$ equip the set $\mathcal{G} / \Sigma$ with the discrete topology and consider the topological space

$$
\widehat{\mathcal{G}} \stackrel{\text { def }}{=} \lim _{\Sigma} \mathcal{G} / \Sigma
$$

where the limit is taken over all congruence subgroups $\Sigma$.

Lemma 3.2 (a) The intersection of all congruence subgroups $N=\bigcap_{\Sigma} \Sigma$ is a normal subgroup of $\mathcal{G}$.

(b) The natural map $p: \mathcal{G} \rightarrow \widehat{\mathcal{G}}$ factors through the injection $\mathcal{G} / N \hookrightarrow \widehat{\mathcal{G}}$ and has dense image.

(c) The group multiplication on $\mathcal{G} / N$ extends by continuity to $\widehat{\mathcal{G}}$ and makes $\widehat{\mathcal{G}}$ a totally disconnected locally compact group.

We call $\widehat{\mathcal{G}}$ the congruence completion of $\mathcal{G}$. Although the notation doesn't reflect this, the completion $\widehat{\mathcal{G}}$ depends on the choice of the Hecke subgroup $\Gamma$. A Hecke subgroup $\Gamma$ is called effective, if the normal subgroup $N$ above is trivial.

Proof: (a) Let $n \in N$ and let $g \in \mathcal{G}$. For a given congruence subgroup $\Sigma$ we have that $n \in \Sigma \cap g^{-1} \Sigma g$, and so $g n g^{-1} \in \Sigma$. As $\Sigma$ varies, we find $g n g^{-1} \in N$. 
(b) Let $g, g^{\prime} \in \mathcal{G}$ with $p(g)=p\left(g^{\prime}\right)$. This means that $g \Sigma=g^{\prime} \Sigma$ for every congruence subgroup and so $g N=g^{\prime} N$. For given $\left(g_{\Sigma}\right)_{\Sigma} \in \widehat{\mathcal{G}}$ the sets $U_{\Sigma}=\left\{h \in \widehat{\mathcal{G}}: h_{\Sigma} \Sigma=g_{\Sigma} \Sigma\right\}$ form a neighborhood base. Clearly the element $g_{\Sigma} \in \mathcal{G}$ is mapped into $U_{\Sigma}$, so the image of $p$ is dense.

(c) Let $\bar{g}=\left(g_{\Sigma}\right)_{\Sigma} \in \widehat{\mathcal{G}}$. Then the net $\left(p\left(g_{\Sigma}\right)\right)_{\Sigma}$ converges to $\bar{g}$. For $\bar{h}=$ $\left(h_{\Sigma}\right) \in \widehat{\mathcal{G}}$ it is easy to see that the net $\left(p\left(g_{\Sigma} h_{\Sigma}\right)\right)_{\Sigma}$ converges in $\overline{\mathcal{G}}$. We call the limit $\bar{g} \bar{h}$. This multiplication has the desired properties.

The initial topology defined by $p$ on $\mathcal{G}$ makes $\mathcal{G}$ a topological group with the congruence groups forming a unit neighborhood base. Clearly every smooth $\overline{\mathcal{G}}$-module is a smooth $\mathcal{G}$-module by restriction. But also the converse is true: Every smooth $\mathcal{G}$-module extends uniquely to a smooth $\overline{\mathcal{G}}$-module and these two operations of restriction and extension are inverse to each other. Let

$$
\widehat{\mathrm{H}}_{q}^{p}(V) \stackrel{\text { def }}{=} \underset{\Sigma}{\lim _{\boldsymbol{\Sigma}}} \mathrm{H}_{q}^{p}(\Sigma, V),
$$

where the limit is taken over all congruence subgroups of $\Gamma$. For $p=0$ we also define

$$
\widehat{\overline{\mathrm{H}}}_{q}^{0}(V)=\underset{\Sigma}{\lim _{\Sigma}} \overline{\mathrm{H}}_{q}^{0}(\Sigma, V) .
$$

Note that $\widehat{\overline{\mathrm{H}}}_{0}^{0}(V)=V^{\infty}$. For $g \in \mathcal{G}$, the map induced by $g$ :

$$
\mathrm{H}_{q}^{p}(\Sigma, V) \rightarrow \mathrm{H}_{q}^{p}\left(g \Sigma g^{-1}, V\right) \stackrel{\text { res }}{\longrightarrow} \mathrm{H}_{q}^{p}\left(\Sigma \cap g \Sigma g^{-1}, V\right)
$$

defines an action of $\mathcal{G}$ on $\widehat{\mathrm{H}}_{q}^{p}(V)$.

Assume from now on that $\Gamma$ is finitely generated and $R$ is a field. Then every finite-index subgroup $\Sigma \subset \Gamma$ is finitely generated as well, so Lemma 1.1 applies to all modules $V$.

Let $R$ be a field and consider the order-lowering map

$$
\overline{\mathrm{H}}_{q}^{0}(\Sigma, V) \hookrightarrow \operatorname{Hom}(\Sigma, R) \otimes \overline{\mathrm{H}}_{q-1}^{0}(\Sigma, V) .
$$

By iteration we get an injection

$$
\overline{\mathrm{H}}_{q}^{0}(\Sigma, V) \hookrightarrow \operatorname{Hom}(\Sigma, R)^{\otimes(q-1)} \otimes V^{\Sigma} .
$$

Taking limits we get an injection

$$
\widehat{\overline{\mathrm{H}}}_{q}^{0}(V) \hookrightarrow\left(\lim _{\vec{\Sigma}} \operatorname{Hom}(\Sigma, R)\right)^{\otimes(q-1)} \otimes V^{\infty} .
$$


Write

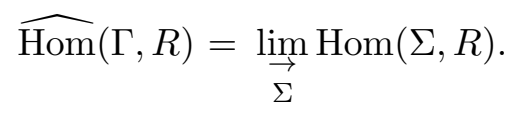

We have shown:

Proposition 3.3 If $\Gamma$ is finitely generated and $R$ is a field, then there is a natural injection of smooth modules

$$
\widehat{\overline{\mathrm{H}}}_{q}^{0}(V) \hookrightarrow \widehat{\operatorname{Hom}}(\Gamma, R)^{\otimes(q-1)} \otimes V^{\infty} .
$$

There are examples when this map is not surjective.

Proposition 3.4 Let $(\mathcal{G}, \Gamma)$ be a Hecke-pair. Assume $\Gamma$ to be finitely generated and let $V$ be an $R[\Gamma]$-module such that

$$
\mathrm{H}^{1}(\Sigma, V)=0
$$

for every congruence subgroup $\Sigma$ of $\Gamma$. Then for every $q \geq 1$ there is an isomorphism of $\mathcal{G}$-modules

$$
\widehat{\overline{\mathrm{H}}}_{q}^{0}(V) \stackrel{\cong}{\longrightarrow} \widehat{\mathrm{H}}_{q-1}^{1}(R) \otimes V^{\infty} .
$$

Proof: This is a consequence of Proposition 2.3.

\section{$4 \quad$ Higher order $L^{2}$-spaces}

Let $G$ be a locally compact group and $\Gamma$ a countable discrete subgroup. We consider the action of $\Gamma$ on $G$ by left translations.

Lemma 4.1 The group $\Gamma$ is closed in $G$. It acts strongly discontinuously in the sense that for every compact subset $C \subset G$ the set

$$
\{\gamma \in \Gamma: C \cap \gamma C \neq \emptyset\}
$$

is finite. 
Proof: It is known that discrete subgroups are closed [3]. Further, discreteness of $\Gamma$ implies that $\Gamma$ meets every compact set in only finitely many points. With $C$ the set $C C^{-1}$ is also compact, which gives the claim.

A fundamental set is a set $F \subset G$ of representatives for the quotient $\Gamma \backslash G$ which is measurable.

A fundamental set $F$ is called locally finite if there exists an open neighborhood $U$ of the closure $\bar{F}$ such that $U$ only meets finitely many translates of $\bar{F}$, i.e., the set

$$
\{\gamma \in \Gamma: \gamma \bar{F} \cap U \neq \emptyset\}
$$

is finite.

Lemma 4.2 Any measurable set $M \subset G$ which contains a set of representatives for $\Gamma \backslash G$, contains a fundamental set.

Proof: According to a theorem of Feldman and Greenleaf [11] there exists a fundamental set $\tilde{F} \subset G$. We now enumerate the elements of $\Gamma$, so

$$
\Gamma=\left\{1=\gamma_{1}, \gamma_{2}, \gamma_{3}, \ldots\right\} .
$$

We set $F_{1}=M \cap \tilde{F}$ and iteratively we define

$$
F_{j+1}=F_{j} \cup\left[\left(M \cap \gamma_{j+1} \tilde{F}\right) \backslash \Gamma M_{j}\right] .
$$

Then $F=\bigcup_{j=1}^{\infty} F_{j}$ is a fundamental set contained in $M$.

Two fundamental sets $F_{1}, F_{2}$ are called compatible, if there is a finite subset $E \subset \Gamma$ such that

$$
F_{1} \subset E F_{2} \quad \text { and } \quad F_{2} \subset E F_{1} .
$$

Let $M$ be the space of all measurable functions modulo nullfunctions on $G$ and let $M_{q}$ be the subspace of all $f \in F$ such that $I^{q} f=0$, where $I$ is the augmentation ideal in $\mathbb{C}[\Gamma]$.

Proposition 4.3 Fix a fundamental set $F \subset G$. Assume that $\Gamma$ is finitely generated and let $S$ be a finite symmetric set of generators of $\Gamma$ which is supposed to contain the unit element. Then any $f \in M_{q}$ is uniquely determined by its restriction to

$$
S^{q-1} F=\bigcup_{s_{1}, \ldots, s_{q-1} \in S} s_{1} \cdots s_{q-1} F
$$


here we interpret $S^{0} F$ as $F$ itself. The space

$$
M_{q} \cap L^{2}\left(S^{q-1} F\right)
$$

does neither depend on the choice of $S$ nor on the choice of $F$ in a given compatibility class. We denote this space by $L_{q}^{2}(F)$ or, if there is a given choice of $F$, by $L_{q}^{2}(\Gamma \backslash X)$.

Also, the topology on $L_{q}^{2}(F)$, given by the $L^{2}$-structure, is independent of the choices in the same way as the space itself.

Proof: We have to show that any $f \in M_{q}$ which vanishes on $S^{q-1} F$, is zero. We use induction on $q$. The case $q=1$ is clear. Let $q \geq 2$ and set $\bar{M}_{q}=M_{q} / M_{q-1}$. Consider the order lowering operator

$$
\Lambda: M_{q} \rightarrow \operatorname{Hom}\left(\Gamma, \bar{M}_{q-1}\right) \cong \operatorname{Hom}(\Gamma, \mathbb{C}) \otimes \bar{M}_{q-1}
$$

given by

$$
\Lambda(f)(\gamma)=(\gamma-1) f
$$

The kernel of $\Lambda$ is $M_{q-1}$. Now assume $f \in M_{q}$ vanishes on $S^{q-1} F$. For every $s \in S$ we have $\left.(s-1) f\right|_{S^{q-1} F}=0$ and hence, by induction hypothesis, $(s-1) f=0$. As $S$ generates $\Gamma$, this means $\Lambda(f)=0$ and so $f \in M_{q-1}$, so again by induction hypothesis we get $f=0$.

We next show

$$
M_{q} \cap L^{2}\left(S^{q} F\right)=M_{q} \cap L^{2}\left(S^{q+j} F\right)
$$

for every $j \geq 0$. The inclusion " $\supset$ " is clear. We show " $\subset$ " by induction on $q$. For $q=1$ the claim is clear. So assume $q \geq 2$ and the claim proven for $q-1$. We show $M_{q} \cap L^{2}\left(S^{q-1+j} F\right) \subset M_{q} \cap L^{2}\left(S^{q+j} F\right)$ for every $j \geq 0$. For this let $f \in M_{q} \cap L^{2}\left(S^{q-1+j} F\right)$ and let $s \in S$. Then $f\left(s^{-1} z\right)=f(z)+$ $f\left(s^{-1} z\right)-f(z)=f(z)+(s-1) f(z)$. The function $f(z)$ is in $L^{2}\left(S^{q-1+j} F\right)$ and the function $(s-1) f(z)$ is in $M_{q-1} \cap L^{2}\left(S^{q-2+j} F\right)$ the latter space equals $M_{q-1} \cap L^{2}\left(S^{q-1+j} F\right)$ by induction hypothesis. It follows that $f\left(s^{-1} z\right)$ is in $L^{2}\left(S^{q-1+j} F\right)$, so $f \in L^{2}\left(s S^{q-1+j} F\right)$. Since this holds for every $s \in S$, we get $f \in L^{2}\left(S^{q+j} F\right)$ as claimed.

From this we conclude the independence of $S$. Let $S_{1}$ be a second finite symmetric set of generators containing the unit. Then there exists $j \geq$ 0 such that $S_{1}^{q} \subset S^{q+j}$. Hence it follows that $M_{q} \cap L^{2}\left(S_{1}^{q-1} F\right) \subset M_{q} \cap$ $L^{2}\left(S^{q-1+j} F\right)=M_{q} \cap L^{2}\left(S^{q-1} F\right)$. By symmetry we get $M_{q} \cap L^{2}\left(S_{1}^{q-1} F\right)=$ $M_{q} \cap L^{2}\left(S^{q-1} F\right)$ as claimed. We have to show independence of the choice of 
$F$. So let $F^{\prime}$ be another fundamental set which is compatible with $F$. Then there exists $j \geq 0$ such that $S^{q} F^{\prime} \subset S^{q+j} F$ and we prove independence as above.

Finally, for the topology, let $\left(f_{\nu}\right)$ be a sequence in $M_{q} \cap L^{2}\left(S^{q-1} F\right)$ tending to zero. We argue as in the proof of $M_{q} \cap L^{2}\left(S^{q-1} F\right)=M_{q} \cap L^{2}\left(S^{q-1+j} F\right)$ to show that $\left(f_{\nu}\right)$ also tends to zero in $L^{2}\left(S^{q-1+j} F\right)$. From here on the proof of independence of choices for the topology on the space $L_{q}^{2}(F)$ proceeds as above.

We will remove the dependence on the choice of a fundamental set by giving a canonical choice (up to compatibility) in the case when $\Gamma \backslash G$ is compact.

Lemma 4.4 There exists a relatively compact fundamental set if and only if $\Gamma \backslash G$ is compact. Each relatively compact fundamental set is locally finite and any two relatively compact fundamental sets are compatible.

Proof: Let $\pi: G \rightarrow \Gamma \backslash G$ be the projection. This map is continuous and open. Let $F$ be a relatively compact fundamental set, then $\Gamma \backslash G$ is the image under $\pi$ of the compact set $\bar{F}$, hence $\Gamma \backslash G$ is compact.

For the converse assume that $\Gamma \backslash G$ is compact. For each $x \in G$ fix a relatively compact open neighborhood $U_{x}$ of $x$. The sets $\pi\left(U_{x}\right)$ form an open covering of $\Gamma \backslash G$, so finitely many suffice. This means there are $x_{1}, \ldots, x_{n} \in G$ such that the compact set $\tilde{F}=\bar{U}_{x_{1}} \cup \cdots \cup \bar{U}_{x_{n}}$ contains a set of representatives. By Lemma 4.2 it contains a fundamental set which is necessarily relatively compact.

For the second assertion let $F$ be a relatively compact fundamental set. Let $U \supset F$ be a relatively compact open neighborhood of $\bar{F}$. As $\Gamma$ acts strongly discontinuously, it follows that $\bar{U}$ meets only finitely many translates of $\bar{F}$ and hence $U$ only meets finitely many translates of $\bar{F}$.

Finally, let $F_{1}, F_{2}$ be two relatively compact fundamental sets. Let $U$ be an open neighborhood of $F_{1}$ that only meets finitely many translates of $F_{1}$. Then the family $(\gamma U)_{\gamma \in \Gamma}$ is an open covering of $G$, so there are $\gamma_{1}, \ldots \gamma_{n}$ such that $\bar{F}_{2} \subset \gamma_{1} U \cup \cdots \cup \gamma_{n} U$. Now $U$ is contained in a finite number of translates of $F_{1}$, so there exists a finite set $E \subset \Gamma$ such that $F_{2} \subset E F_{1}$. By symmetry, we get the other direction, too.

From now on we assume that $\Gamma$ is cocompact, i.e. $\Gamma \backslash G$ is compact. In this case we say that $\Gamma$ is a uniform lattice in $G$. We consider the space $L_{\text {loc }}^{2}(G)$ 
of all locally square-integrable functions on $G$ (modulo nullfunctions). So we have $f \in L_{\text {loc }}^{2}(G)$ if for every $x \in G$ there exists an open neighborhood $U$ such that $\left.f\right|_{U} \in L^{2}(U)$. This is equivalent to saying that $\left.f\right|_{K} \in L^{2}(K)$ for every compact subset $K \subset G$. We have

$$
L_{q}^{2}(\Gamma \backslash G)=H_{q}^{0}\left(\Gamma, L_{\mathrm{loc}}^{2}(G)\right)
$$

Definition 4.5 The elements of $L_{q}^{2}(\Gamma \backslash G)$ will be called automorphic forms of order $q$. Automorphic forms of order 1 are classical automorphic forms. Those of order $\geq 2$ are also referred to as automorphic forms of higher order.

Proposition 4.6 For every $q \geq 0$ we have

$$
H_{q}^{1}\left(\Gamma, L_{\mathrm{loc}}^{2}(G)\right)=0 .
$$

Proof: Let $F$ be a relatively compact fundamental set. By Lemma 2.1 (b) it suffices to consider the case $q=0$. Let $\alpha: \Gamma \rightarrow L_{\text {loc }}^{2}(G)$ be a 1-cocycle, i.e. a map that satisfies $\alpha(\gamma \tau)=\gamma \alpha(\tau)+\alpha(\gamma)$. We set

$$
f(x)=\sum_{\tau \in \Gamma} \alpha(\tau)(x) \mathbf{1}_{F}\left(\tau^{-1} x\right) .
$$

A simple computation shows $(1-\gamma) f=\alpha(\gamma)$ and since $\alpha \in L_{\text {loc }}^{2}(G)$ it follows that $f \in L_{\mathrm{loc}}^{2}(G)$. The proposition follows.

Proposition 4.7 For every $q \geq 1$ there is a natural exact sequence of continuous linear maps,

$$
0 \rightarrow L_{q}^{2}(\Gamma \backslash G) \rightarrow L_{q+1}^{2}(\Gamma \backslash G) \rightarrow L^{2}(\Gamma \backslash G)^{N_{\Gamma}(q)} \rightarrow 0 .
$$

Proof: This follows from Lemma 2.1 together with Proposition 4.6.

\section{$5 \quad$ Lie groups}

In this section $G$ will be a Lie group. In this case we write

$$
C_{q}^{\infty}(\Gamma \backslash G)=H_{q}^{0}\left(\Gamma, C^{\infty}(G)\right) .
$$


Proposition 5.1 For a Lie group $G$ and a discrete subgroup $\Gamma$ we have

$$
H^{1}\left(\Gamma, C^{\infty}(G)\right)=0 .
$$

Consequently, for every $q \geq 1$ there is a natural exact sequence

$$
0 \rightarrow C_{q}^{\infty}(\Gamma \backslash G) \rightarrow C_{q+1}^{\infty}(\Gamma \backslash G) \rightarrow C^{\infty}(\Gamma \backslash G)^{N_{\Gamma}(q)} \rightarrow 0 .
$$

Proof: This is a a part of Proposition 2.3.2 in [6].

Let $\pi \in \hat{G}$. A representation $\left(\beta, V_{\beta}\right)$ of $G$ is said to be of type $\pi$, if it is of finite length and every irreducible subquotient is isomorphic to $\pi$. For a representation $\left(\eta, V_{\eta}\right)$ we define the $\pi$-isotype as

$$
V_{\eta}(\pi) \stackrel{\text { def }}{=} \sum_{\substack{V_{\beta} \subset V_{\eta} \\ \text { of type } \pi}} V_{\beta},
$$

where the sum runs over all subrepresentations $V_{\beta}$ of type $\pi$. If $\pi \neq \pi^{\prime}$, then $V_{\eta}(\pi) \cap V_{\eta}\left(\pi^{\prime}\right)=0$.

If $\Gamma$ is a uniform lattice, then the representation of $G$ on $L^{2}(\Gamma \backslash G)$ is a direct sum of irreducible representations, each occuring with finite multiplicity, i.e.,

$$
L^{2}(\Gamma \backslash G) \cong \bigoplus_{\pi \in \widehat{G}} m_{\Gamma}(\pi) \pi,
$$

with $m_{\Gamma}(\pi) \in \mathbb{N}_{0}$.

Theorem 5.2 (Spectral decomposition) Let $G$ be a semisimple Lie group and $\Gamma$ a uniform lattice in $G$. We write $V_{q}=\mathrm{H}_{q}^{0}(\Gamma, \Omega)$, where $\Omega=L_{\text {loc }}^{2}(G)$ or $\Omega=C^{\infty}(G)$. For every $q \geq 1$ there is an isotypical decomposition

$$
V_{q}=\varlimsup_{\pi \in \hat{G}_{\Gamma}} V_{q}(\pi),
$$

and each $V_{q}(\pi)$ is of type $\pi$ itself. The exact sequence of Proposition 4.7 induces an exact sequence

$$
0 \rightarrow V_{q}(\pi) \rightarrow V_{q+1}(\pi) \rightarrow V_{1}(\pi)^{m_{\Gamma}(\pi) N_{\Gamma}(q)} \rightarrow 0
$$

for every $\pi \in \hat{G}$.

Proof: For $\Omega=C^{\infty}(G)$ this is Theorem 2.3.5 of [6]. For $\Omega=L_{\text {loc }}^{2}(G)$ the result follows from the latter by density arguments. 


\section{Sheaf cohomology}

Let $Y$ be a topological space which is path-connected and locally simply connected. Let $\Gamma$ be the fundamental group of $Y$ and let $X \stackrel{\pi}{\longrightarrow} Y$ be the universal covering.

For a sheaf $\mathcal{F}$ on $Y$ define

$$
H_{q}^{0}(Y, \mathcal{F}) \stackrel{\text { def }}{=} H_{q}^{0}\left(\Gamma, H^{0}\left(X, \pi^{*} \mathcal{F}\right)\right) .
$$

Let $\operatorname{Mod}(R)$ be the category of $R$-modules, let $\operatorname{Mod}_{R}(Y)$ be the category of sheaves of $R$-modules on $Y$, and let $\operatorname{Mod}_{R}(X)_{\Gamma}$ be the category of sheaves over $X$ with an equivariant $\Gamma$-action. Then $\mathrm{H}_{q}^{0}(Y, \cdot)$ is a left exact functor from $\operatorname{Mod}_{R}(Y)$ to $\operatorname{Mod}(R)$. We denote its right derived functors by $\mathrm{H}_{q}^{p}(Y, \cdot)$ for $p \geq 0$.

Proposition 6.1 Assume that the universal cover $X$ is contractible.

(a) For each $p \geq 0$ one has a natural isomorphism $\mathrm{H}_{1}^{p}(Y, \mathcal{F}) \cong \mathrm{H}^{p}(Y, \mathcal{F})$.

(b) If a sheaf $\mathcal{F}$ is $\mathrm{H}^{0}(Y, \cdot)$-acyclic, then it is $\mathrm{H}_{q}^{0}(Y, \cdot)$-acyclic for every $q \geq 1$.

Note that part (b) allows one to use flabby or fine resolutions to compute higher order cohomology.

Proof: We decompose the functor $\mathrm{H}_{q}^{0}(Y, \cdot)$ as a composition of functors

$$
\operatorname{Mod}_{R}(Y) \stackrel{\pi^{*}}{\longrightarrow} \operatorname{Mod}_{R}(X)_{\Gamma} \stackrel{\mathrm{H}^{0}(X, \cdot)}{\longrightarrow} \operatorname{Mod}(R[\Gamma]) \stackrel{\mathrm{H}_{q}^{0}(\Gamma, \cdot)}{\longrightarrow} \operatorname{Mod}(R) .
$$

The functor $\pi^{*}$ is exact and maps injectives to injectives. We claim that $\mathrm{H}^{0}(X, \cdot)$ has the same properties. For the exactness, consider the commutative diagram

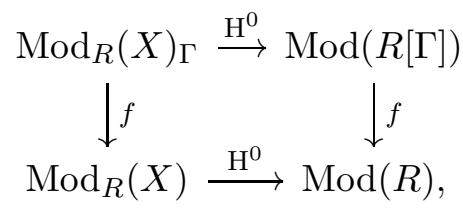

where the vertical arrows are the forgetful functors. As $X$ is contractible, the functor $\mathrm{H}^{0}$ below is exact. The forgetful functors have the property, that a sequence upstairs is exact if and only if its image downstairs is exact. This implies that the above $\mathrm{H}^{0}$ is exact. It remains to show that $\mathrm{H}^{0}$ maps 
injective objects to injective objects. Let $\mathcal{J} \in \operatorname{Mod}_{R}(X)_{\Gamma}$ be injective and consider a diagram with exact row in $\operatorname{Mod}(R[\Gamma])$,

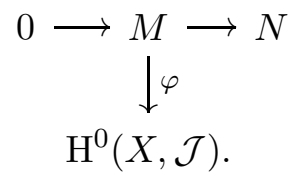

The morphism $\varphi$ gives rise to a morphism $\phi: M \times X \rightarrow \mathcal{J}$, where $M \times X$ stands for the constant sheaf with stalk $M$. Note that $\mathrm{H}^{0}(X, \phi)=\varphi$. As $\mathcal{J}$ is injective, there exists a morphism $\psi: N \times X \rightarrow \mathcal{J}$ making the diagram

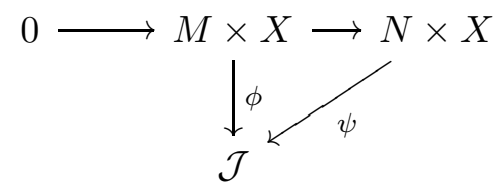

commutative. This diagram induces a corresponding diagram on the global sections, which implies that $\mathrm{H}^{0}(X, \mathcal{J})$ is indeed injective.

For a sheaf $\mathcal{F}$ on $Y$ it follows that

$$
\mathrm{H}^{p}(Y, \mathcal{F})=R^{p}\left(\mathrm{H}^{0}(Y, \mathcal{F})\right)=R^{p} \mathrm{H}_{q}^{0}(\Gamma, \mathcal{F}) \circ \mathrm{H}_{\Gamma}^{0} \circ \pi^{*}=\mathrm{H}_{q}^{p}(Y, \mathcal{F}) .
$$

Now let $\mathcal{F}$ be acyclic. Then we conclude $\mathrm{H}_{0}^{p}(Y, \mathcal{F})=0$ for every $p \geq 1$, so the $\Gamma$-module $V=\mathrm{H}^{0}\left(X, \pi^{*} \mathcal{F}\right)$ is $\Gamma$-acyclic. The claim follows from Lemma 2.1 .

Let $Z$ be a closed subset of $Y$ and let $U$ be its complement. Let $i: Z \hookrightarrow X$ and $j: U \hookrightarrow X$ denote the inclusions.

Proposition 6.2 Let $\mathcal{F}$ be a sheaf on $Y$. The exact sequence

$$
0 \rightarrow j_{!}\left(\left.\mathcal{F}\right|_{U}\right) \rightarrow \mathcal{F} \rightarrow i_{*}\left(\left.\mathcal{F}\right|_{Z}\right) \rightarrow 0
$$

gives rise to a long exact sequence of cohomology groups:

$$
\begin{aligned}
0 \rightarrow \mathrm{H}_{q, c}^{0}(U, \mathcal{F}) \rightarrow & \mathrm{H}_{q}^{0}(Y, \mathcal{F}) \rightarrow \mathrm{H}_{q}^{0}(Z, \mathcal{F}) \\
\rightarrow & \mathrm{H}_{q, c}^{1}(U, \mathcal{F}) \rightarrow \mathrm{H}_{q}^{1}(Y, \mathcal{F}) \rightarrow \mathrm{H}_{q}^{1}(Z, \mathcal{F}) \\
& \quad \cdots \rightarrow \mathrm{H}_{q, c}^{p}(U, \mathcal{F}) \rightarrow \mathrm{H}_{q}^{p}(Y, \mathcal{F}) \rightarrow \mathrm{H}_{q}^{p}(Z, \mathcal{F}) \rightarrow \ldots
\end{aligned}
$$

Here $\mathrm{H}_{q, c}^{0}(U, \mathcal{F})$ stands for the group of all sections in $\mathrm{H}_{q}^{0}(Y, \mathcal{F})$ whose support is contained in $\pi^{-1}(U)$ and $\mathrm{H}_{q}^{p}(Y, \mathcal{F})$ for $p \geq 1$ is the corresponding derived functor. 
Proof: It is easy to see that $\mathrm{H}_{q}^{0}\left(Y, j_{!}\left(\left.\mathcal{F}\right|_{U}\right)\right)=\mathrm{H}_{q, c}^{0}(U, \mathcal{F})$. The functor $\mathcal{F} \mapsto j_{!}\left(\left.\mathcal{F}\right|_{U}\right)$ is exact and maps products of injective skyscraper sheaves to products of injective skyscraper sheaves. As any sheaf has a resolution consisting of products of injective skyscraper sheaves (which are injective themselves), it follows that $\mathrm{H}_{q}^{p}\left(Y, j_{!}\left(\left.\mathcal{F}\right|_{U}\right)\right)=\mathrm{H}_{q, c}^{p}(U, \mathcal{F})$ holds for every $p \geq$ 0 . This proves the assertion.

Assume that $X$ is contractible. Then $Y$ is a classifying space for the group $\Gamma$. So any $R[\Gamma]$-module $V$ gives rise to a locally constant sheaf $\mathcal{V}$ of $R$-modules over $Y$ such that $H^{p}(\Gamma, V) \cong H^{p}(Y, \mathcal{V})$ holds for every $p \geq 0$.

Proposition 6.3 We have natural isomorphisms

$$
\mathrm{H}_{q}^{p}(\Gamma, V) \cong \mathrm{H}_{q}^{p}(Y, \mathcal{V})
$$

for all $p \geq 0, q \geq 1$.

Proof: Fix $q \geq 1$. The functors $V \mapsto \mathrm{H}_{q}^{p}(\Gamma, V)$ form a universal $\delta$-functor on $\operatorname{Mod}(R[\Gamma])$. Let $S h(Y)$ be the category of sheaves of $R$-modules over $Y$, so $\mathcal{F} \mapsto \mathrm{H}_{q}^{p}(Y, \mathcal{F})$ is a universal $\delta$-functor on $S h(Y)$. The functor of sheafification Sheaf $: \operatorname{Mod}(R[\Gamma]) \rightarrow \operatorname{Sh}(Y)$, which to a module $V$ attaches the locally constant sheaf $\mathcal{V}=\operatorname{Sheaf}(V)$, is exact. So $V \mapsto \mathrm{H}_{q}^{p}(Y$, $\operatorname{Sheaf}(V))$ is a $\delta$-functor on $\operatorname{Mod}(R[\Gamma])$. We have to show universality, which we do as usual by showing erasability of $\mathrm{H}^{p}$ for $p \geq 1$. For $V \in \operatorname{Mod}(R[\Gamma])$ let

$$
I V=\{\alpha: \Gamma \rightarrow V\}
$$

the module of all maps from $\Gamma$ to $M$. This is a $\Gamma$-module via

$$
\gamma . \alpha(\tau)=\gamma\left(\alpha\left(\gamma^{-1} \tau\right)\right)
$$

Mapping $v \in V$ to the constant map $v$, one gets an embedding $V \hookrightarrow I V$. By Proposition 6.1 we have to show

$$
\mathrm{H}^{p}(Y, \operatorname{Sheaf}(I V))=0
$$

for $p \geq 1$. Let $\pi: X \rightarrow Y$ be the projection. Then

$$
\operatorname{Sheaf}(I V)=\pi_{*} \tilde{V}
$$

where $\tilde{V}$ stands for the constant sheaf $V$ on $X$. We now show, that $\pi_{*} \tilde{V}$ is acyclic. To this end we resolve $\tilde{V}$ with special acyclic sheaves, which 
are products of skyscraper sheaves with injective stalks. The images under $\pi_{*}$ of these are again products of skyscraper sheaves with injective stalks, hence acyclic. The global sections above and below are the same, so the cohomologies agree, i.e., we have

$$
\mathrm{H}^{p}\left(Y, \pi_{*} \tilde{V}\right)=\mathrm{H}^{p}(X, \tilde{V}) .
$$

As $X$ is contractible, the right hand side is zero.

\section{Cohomology of lattices in Lie groups}

In this section we set $R=\mathbb{C}$. Let $G$ be a semisimple Lie group with compact center and finitely many connected components. Let $\Gamma \subset G$ be a uniform lattice which is torsion-free. Let $Y=\Gamma \backslash X$, then $\Gamma$ is the fundamental group of the manifold $Y$, and the universal covering $X$ of $Y$ is contractible. This means that we can apply the results of the last section. Compare the next result to the classical case [17].

Theorem 7.1 Let $(\sigma, E)$ be a finite dimensional irreducible representation of $G$. There is a natural isomorphism

$$
\begin{aligned}
\mathrm{H}_{q}^{p}(\Gamma, E) \cong \mathrm{H}^{p}\left(\mathfrak{g}, K, \mathrm{H}_{q}^{0}\left(\Gamma, C^{\infty}(G)\right) \otimes E\right) \\
=\bigoplus_{\substack{\pi \in \hat{G} \\
\chi_{\pi}=\chi_{E^{*}}}} \mathrm{H}^{p}\left(\mathfrak{g}, K, C_{q}^{\infty}(\Gamma \backslash G)(\pi) \otimes E\right),
\end{aligned}
$$

where $\mathrm{H}(\mathfrak{g}, K,$.$) denotes (\mathfrak{g}, K)$-cohomology and the direct sum is finite.

Proof: Let $\mathcal{F}_{E}$ be the locally constant sheaf on $Y$ corresponding to $E$. Let $\Omega_{Y}^{p}$ be the sheaf of complex valued $p$-differential forms on $Y$. Then $\Omega_{Y}^{p} \otimes \mathcal{F}_{E}$ is the sheaf of $\mathcal{F}_{E}$-valued differential forms. These form a fine resolution of $\mathcal{F}_{E}$ :

$$
0 \rightarrow \mathcal{F}_{E} \rightarrow \mathbb{C}^{\infty} \otimes \mathcal{F}_{E} \stackrel{d \otimes 1}{\longrightarrow} \Omega_{Y}^{1} \otimes \mathcal{F}_{E} \rightarrow \ldots
$$

Since $\pi^{*} \Omega_{Y}^{\bullet}=\Omega_{X}^{\bullet}$, we conclude that $\mathrm{H}_{q}^{p}(\Gamma, E)$ is the cohomology of the complex $\mathrm{H}_{q}^{0}\left(\Gamma, \mathrm{H}^{0}\left(X, \Omega_{X}^{\bullet} \otimes E\right)\right)$. Let $\mathfrak{g}$ and $\mathfrak{k}$ be the Lie algebras of $G$ and $K$ respectively, and let $\mathfrak{g}=\mathfrak{k} \oplus \mathfrak{p}$ be the Cartan decomposition. Then $\mathrm{H}^{0}\left(X, \Omega^{p} \otimes \mathcal{F}_{E}\right)=\left(C^{\infty}(G) \otimes \bigwedge^{p} \mathfrak{p}\right)^{K} \otimes E$. Mapping a form $\omega$ in this space to $\left(1 \otimes x^{-1}\right) \omega(x)$ one gets an isomorphism to $\left(C^{\infty}(G) \otimes \bigwedge^{p} \mathfrak{p} \otimes E\right)^{K}$, where 
$K$ acts diagonally on all factors and $\Gamma$ now acts on $C^{\infty}(G)$ alone. The first claim follows. For the second, we replace $C_{q}^{\infty}(\Gamma \backslash G)$ by the sum of its isotypes according to Theorem 5.2. The direct sum can be pulled out of cohomology to get

$$
\mathrm{H}_{q}^{p}(\Gamma, E) \cong \bigoplus_{\pi \in \widehat{G}} \mathrm{H}\left(\mathfrak{g}, K, V_{q}(\pi) \otimes E\right),
$$

where $V_{q}=C_{q}^{\infty}(\Gamma \backslash G)$. For each $\pi$ there is a $G$-stable finite filtration

$$
0=F_{0} \subset \cdots \subset F_{n}-V_{q}(\pi)
$$

such that $F_{j} / F_{j-1} \cong \pi^{\infty}$. The exact sequence

$$
0 \rightarrow F_{j-1} \rightarrow F_{j} \rightarrow \pi^{\infty} \rightarrow 0
$$

induces a long exact cohomology sequence

$$
\begin{aligned}
0 & \rightarrow \mathrm{H}^{0}\left(\mathfrak{g}, K, F_{j-1} \otimes E\right) \rightarrow \mathrm{H}^{0}\left(\mathfrak{g}, K, F_{j} \otimes E\right) \rightarrow \mathrm{H}^{0}\left(\mathfrak{g}, K, \pi^{\infty} \otimes E\right) \rightarrow \\
& \rightarrow \mathrm{H}^{1}\left(\mathfrak{g}, K, F_{j-1} \otimes E\right) \rightarrow \mathrm{H}^{1}\left(\mathfrak{g}, K, F_{j} \otimes E\right) \rightarrow \mathrm{H}^{1}\left(\mathfrak{g}, K, \pi^{\infty} \otimes E\right) \rightarrow \\
\cdots & \rightarrow \mathrm{H}^{p}\left(\mathfrak{g}, K, F_{j-1} \otimes E\right) \rightarrow \mathrm{H}^{p}\left(\mathfrak{g}, K, F_{j} \otimes E\right) \rightarrow \mathrm{H}^{p}\left(\mathfrak{g}, K, \pi^{\infty} \otimes E\right) \rightarrow \ldots
\end{aligned}
$$

Now assume $\chi_{\pi} \neq \chi_{E^{*}}$. By Theorem 4.1 of [1] we conclude $\mathrm{H}^{p}\left(\mathfrak{g}, K, \pi^{\infty} \otimes\right.$ $E)=0$ for all $p$. By induction on $j$ one gets $\mathrm{H}^{p}\left(\mathfrak{g}, K, F_{j} \otimes E\right)=0$ for all $p$ and all $j$. Hence in the above sum only those finitely many summands with $\chi_{\pi}=\chi_{E^{*}}$ remain.

Taking $q$-th powers gives a natural surjective map

$$
\left(I / I^{2}\right)^{\otimes q} \rightarrow I^{q} / I^{q+1} .
$$

This dualizes to an injection

$$
\operatorname{Hom}_{\mathbb{C}}\left(I^{q} / I^{q+1}, \mathbb{C}\right) \hookrightarrow \operatorname{Hom}_{\mathbb{C}}\left(I / I^{2}, \mathbb{C}\right)^{\otimes q} .
$$

For $p=1$ and $q \geq 1$ we thus get,

$$
\begin{aligned}
\mathrm{H}_{q-1}^{1}(\Gamma, \mathbb{C}) & \cong \operatorname{Hom}_{\mathbb{C}}\left(I^{q} / I^{q+1}, \mathbb{C}\right) \\
& \cong \operatorname{Hom}_{\mathbb{C}}\left(I / I^{2}, \mathbb{C}\right)^{\otimes q} \\
& \cong \mathrm{H}^{1}(\Gamma, \mathbb{C})^{\otimes q} \\
& \cong\left(\bigoplus_{\substack{\pi \in \widehat{G} \\
\chi_{\pi}=\chi_{\mathbb{C}}}} m_{\Gamma}(\pi) \mathrm{H}^{1}\left(\mathfrak{g}, K, \pi^{\infty}\right)\right)^{\otimes q}
\end{aligned}
$$




\section{Arithmetic groups}

In this section we put $R=\mathbb{C}$. Let $\mathbb{G}$ be a linear algebraic group over $\mathbb{Q}$ which is simple and simply connected and such that $G=\mathbb{G}(\mathbb{R})$ has no compact component. By strong approximation, the group $\mathcal{G}=\mathbb{G}(\mathbb{Q})$ is dense in $\mathbb{G}\left(\mathbb{A}_{\text {fin }}\right)$, where $\mathbb{A}_{\text {fin }}$ is the ring of finite adeles. Let $K_{\text {fin }}$ be a given compact open subgroup of $\mathbb{G}\left(\mathbb{A}_{\text {fin }}\right)$ and let $\Gamma=\mathcal{G} \cap K_{\text {fin }}$. Then $(\mathcal{G}, \Gamma)$ is a Hecke-pair and $\Gamma$ can be chosen to be effective. In this case the congruence completion is $\overline{\mathcal{G}} \cong \mathbb{G}\left(\mathbb{A}_{\text {fin }}\right)$. Let $\widehat{\mathbb{G}(\mathbb{A})}$ be the unitary dual of $\mathbb{G}(\mathbb{A})$. Note that every $\pi \in \widehat{\mathbb{G}(\mathbb{A})}$ is a tensor product

$$
\pi=\left(\bigotimes_{p} \pi_{p}\right) \otimes \pi_{\infty}
$$

where the product runs over all primes $p$ and $\pi_{p} \in \widehat{\mathbb{G}_{\left(\mathbb{Q}_{p}\right)}}$. We also denote the representation $\bigotimes_{p} \pi_{p}$ of the group $\mathbb{G}\left(\mathbb{A}_{\mathrm{fin}}\right)$ by $\pi_{\mathrm{fin}}$.

Let $(\pi, V)$ be a representation of the locally compact group $\mathbb{G}\left(\mathbb{A}_{\text {fin }}\right)$. A vector $v \in V$ is called smooth, if $v$ is stabilized by an open subgroup of $\mathbb{G}\left(\mathbb{A}_{\text {fin }}\right)$. For any continuous representation, the set $V^{\infty}$ of smooth vectors is a dense, $\mathbb{G}\left(\mathbb{A}_{\text {fin }}\right)$-stable subspace.

In this paper we assume that

$$
\mathbb{Q}-\operatorname{rank}(\mathbb{G})=0
$$

this implies that $\mathbb{G}(\mathbb{Q}) \backslash \mathbb{G}(\mathbb{A})$ is compact. For $\pi \in \widehat{\mathbb{G}(\mathbb{A})}$ let

$$
m(\pi)=\operatorname{dim} \operatorname{Hom}_{\mathbb{G}(\mathbb{A})}\left(V_{\pi}, L^{2}(\mathbb{G}(\mathbb{Q}) \backslash \mathbb{G}(\mathbb{A}))\right) .
$$

Then $m(\pi) \in \mathbb{N}_{0}$ and

$$
L^{2}(\mathbb{G}(\mathbb{Q}) \backslash \mathbb{G}(\mathbb{A})) \cong \bigoplus_{\pi \in \mathbb{G}(\mathbb{A})} m(\pi) \pi,
$$

Fix a congruence subgroup $\Sigma$ of $\Gamma$. Then there exists a compact open subgroup $K_{\Sigma}$ of $\mathbb{G}\left(\mathbb{A}_{\text {fin }}\right)$ such that $\Sigma=K_{\Sigma} \cap \mathbb{G}(\mathbb{Q})$. The $G$-equivariant identification $\mathbb{G}(\mathbb{Q}) \backslash \mathbb{G}(\mathbb{A}) / K_{\Sigma} \cong \Sigma \backslash G$ gives an isomorphism of unitary $G$ representations

$$
L^{2}(\mathbb{G}(\mathbb{Q}) \backslash \mathbb{G}(\mathbb{A}))^{K_{\Sigma}} \cong L^{2}(\Sigma \backslash G) .
$$


Hence we get

$$
L^{2}(\Sigma \backslash G) \cong \bigoplus_{\pi_{\infty} \in \widehat{G}} m_{\Sigma}\left(\pi_{\infty}\right) \pi_{\infty}
$$

where

$$
m_{\Sigma}\left(\pi_{\infty}\right)=\sum_{\left.\pi_{\text {fin }} \in \mathbb{G}_{\left(\mathbb{A}_{\text {fin }}\right.}\right)} m\left(\pi_{\text {fin }} \otimes \pi_{\infty}\right) \operatorname{dim}\left(\pi_{\text {fin }}^{K_{\Sigma}}\right)
$$

For $\pi \in \widehat{G}$ we write

$$
h^{1}(\pi)=\operatorname{dim} \mathrm{H}^{1}\left(\mathfrak{g}, K, \pi^{\infty}\right) \in \mathbb{N}_{0} .
$$

We have shown that for each congruence subgroup $\Sigma$ there is a natural inclusion

$$
\mathrm{H}_{q}^{1}(\Sigma, \mathbb{C}) \hookrightarrow\left(\bigoplus_{\pi \in \widetilde{G(\mathbb{A})}} m(\pi) h^{1}(\pi) \pi_{\mathrm{fin}}^{K_{\Sigma}}\right)^{\otimes q} .
$$

Taking limits we see that there is a natural map of $\mathbb{G}\left(\mathbb{A}_{\text {fin }}\right)$-representations

$$
\widehat{\mathrm{H}}_{q}^{1}(\mathbb{C}) \hookrightarrow\left(\bigoplus_{\pi \in \widehat{\mathbb{G}(\mathbb{A})}} m(\pi) h^{1}(\pi) \pi_{\mathrm{fin}}\right)^{\otimes q}
$$

We now state the main theorem.

Theorem 8.1 (a) Let $q \geq 1$. The $\mathbb{G}\left(\mathbb{A}_{\text {fin }}\right)$-representation on $\widehat{\mathrm{H}}_{q}^{1}(\mathbb{C})$ is the space of smooth vectors of a unitary representation $\widehat{\mathrm{H}}_{q}^{1}(\mathbb{C})^{u}$ which is a subrepresentation of the tensor power

$$
\left(\bigoplus_{\pi \in \widetilde{\mathbb{G}(\mathbb{A})}} m(\pi) h^{1}(\pi) \pi_{\text {fin }}\right)^{\otimes q} \cong \mathrm{H}^{1}\left(\mathfrak{g}, K, L^{2}(\mathbb{G}(\mathbb{Q}) \backslash \mathbb{G}(\mathbb{A}))\right)^{\otimes q}
$$

(b) The $\mathbb{G}(\mathbb{A})$-representation on $\hat{\bar{H}}_{q}^{0}\left(L_{\text {loc }}^{2}(G)\right)$ is the space of $\mathbb{G}\left(\mathbb{A}_{\text {fin }}\right)$-smooth vectors of a unitary representation which is isomorphic to

$$
\widehat{\mathrm{H}}_{q}^{1}(\mathbb{C})^{u} \otimes L^{2}(\mathbb{G}(\mathbb{Q}) \backslash \mathbb{G}(\mathbb{A})) .
$$

Here $\mathbb{G}\left(\mathbb{A}_{\mathrm{fin}}\right)$ acts on both tensor factors, but $G=\mathbb{G}(\mathbb{R})$ acts only on the second.

Proof: Part (a) has been shown in this section above and part (b) is then a direct application of Proposition 3.4. 


\section{References}

[1] Borel, A.; Wallach,N.: Continuous Cohomology, Discrete Groups, and Representations of Reductive Groups. Ann. Math. Stud. 94, Princeton 1980.

[2] Chinta, G.; Diamantis, N.; O'Sullivan, C.: Second order modular forms. Acta Arith. 103 (2002), no. 3, 209-223

[3] Deitmar, Anton; Echterhoff, Siegfried: Principles of Harmonic Analysis. Springer-Verlag 2008.

[4] Deitmar, A.: Higher order group cohomology and the EichlerShimura map. J. reine u. angew. Math. 629, 221-235 (2009).

[5] Deitmar, A.; Diamantis, N.: Automorphic forms of higher order. Journal of the LMS. 80, 18-34 (2009).

[6] Deitmar, A.: Higher order invariants, cohomology, and automorphic forms. http://arxiv.org/abs/0811.1088.

[7] Diamantis, N.; Knopp, M.; Mason, G.; O'Sullivan, C.: Lfunctions of second-order cusp forms. Ramanujan J. 12 (2006), no. $3,327-347$.

[8] Diamantis, N.; O'Sullivan, C.: The dimensions of spaces of holomorphic second-order automorphic forms and their cohomology. Trans. Amer. Math. Soc. 360, no. 11, 5629-5666 (2008).

[9] Diamantis, N.; Sim, D.: The classification of higher-order cusp forms. J. Reine Angew. Math. 622, 121-153 (2008).

[10] Farmer, D.: Converse theorems and second order modular forms. AMS Sectional Meeting. Salt Lake City (2002).

[11] Feldman, J.; Greenleaf, F. P.: Existence of Borel transversals in groups. Pacific J. Math. 251968 455-461.

[12] Goldfeld, D.; Gunnells, P.: Eisenstein series twisted by modular symbols for the group $\mathrm{SL}_{n}$. Math. Res. Lett. 7, no. 5-6, 747-756 (2000). 
[13] Goldfeld, D.: Modular forms, elliptic curves and the $A B C$ conjecture. A panorama of number theory or the view from Baker's garden (Zürich, 1999), 128-147, Cambridge Univ. Press, Cambridge, 2002 .

[14] Imamoglu, Ö.; O'Sullivan, C.: Parabolic, hyperbolic and elliptic Poincaré series. Acta Arith. 139, no. 3, 199-228 (2009).

[15] Imamoglu, Ö.; Martin, Y.: A converse theorem for second-order modular forms of level N. Acta Arith. 123 (2006), no. 4, 361-376.

[16] Kleban, P.; Zagier, D.: Crossing probabilities and modular forms. J. Statist. Phys. 113 (2003), no. 3-4, 431-454

[17] Schwermer, J.: Cohomology of arithmetic groups, automorphic forms and L-functions. Cohomology of arithmetic groups and automorphic forms (Luminy-Marseille, 1989), 1-29, Lecture Notes in Math., 1447, Springer, Berlin, 1990.

Mathematisches Institut

Auf der Morgenstelle 10

72076 Tübingen

Germany

deitmar@uni-tuebingen.de

13.11.2018 